\title{
Redundancy masking and the identity crowding debate
}

\author{
(John) Henry Taylor (1) | Bilge Sayim
}

University of Birmingham,

Edgbaston, UK

\section{Correspondence}

(John) Henry Taylor, ERI Building,

University of Birmingham, Edgbaston

B15 2TT, UK.

Email: j.h.taylor.1@bham.ac.uk

\section{Funding information}

Schweizerischer Nationalfonds zur

Förderung der Wissenschaftlichen

Forschung, Grant/Award Number:

PP00P1_163723

\begin{abstract}
Some have claimed that identity crowding offers a case of conscious perception of an object to which we are unable to pay attention. Opponents of this view offer alternative explanations, which emphasise the importance of prior knowledge, amongst other factors. We review new empirical evidence showing that prior knowledge has a profound effect on identity crowding. We argue that this is problematic for the "conscious seeing without attention" view, and supports an opposing view.
\end{abstract}

\section{K E Y W O R D S}

attention, block, consciousness, crowding, redundancy masking

\section{1 | IDENTITY CROWDING}

If you fixate your gaze at the top "+" in Figure 1, you will probably find it easy to discern the letter on the right. Now fixate the middle "+." You will find it harder to identify the middle letter on the right. This is crowding: when an item in peripheral vision is flanked by other items, it becomes harder to identify. Identity crowding occurs when the target (the middle letter) and the flankers (the outer letters) are identical (bottom row). Ned Block has claimed that, in identity crowding, the target is consciously seen, but that it escapes attention (Block, 2012). ${ }^{1}$

Block's argument for the claim that we consciously see the target is that we are able to detect it, differentiate it from the background, discriminate it, identify it, and have demonstrative thoughts about it, and thus "it is difficult to see a rationale for denying that one can consciously see it" (Block, 2012, pp. 175 and 177). This argument will be our focus.

Block draws on an operationalist definition of attention (Cavanagh, He, \& Intriligator, 1999) in order to argue that the middle letter is unattended. Assessment of this claim would require a thorough evaluation of this definition of attention. We do not have space for this, and so we will 


\section{$+$

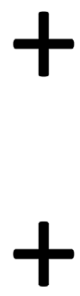

$\mathrm{T}$

FIGURE 1 The top is the non-crowded case, the middle is "normal" crowding and on the bottom is identity crowding
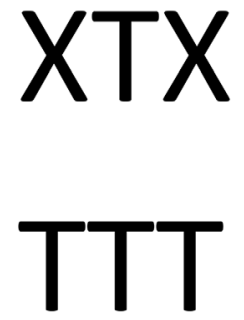

grant this claim for the sake of argument (Prettyman, 2018; Taylor \& Sayim, 2018, p. 22, Taylor, 2018). We also will not discuss Block's appeals to summary statistics (Block, 2013), his claims about natural kinds (Block, 2012, 2013), or his appeals to Freeman and Pelli (2007). These arguments have been extensively discussed elsewhere (Richards, 2016; Taylor \& Sayim, 2018).

\section{2 | PRIOR KNOWLEDGE AND THE HYBRID VIEW}

Taylor and Sayim (2018) propose a view on which we consciously see the group as a whole, but do not consciously see the target (they grant that subjects cannot attend the target). The view then cites a variety of factors, which are jointly sufficient to explain subjects' (apparent) abilities to discriminate, differentiate, identify and have thoughts about the middle letter. Call this the hybrid view. The factors include:

i. Flanker substitution: subjects mistake a flanker for the target, which goes unnoticed because the flankers are the same as the target.

ii. Macroscopic properties of the group as a whole: the appearance of the whole group as a repeating regular pattern, indicates that there was more than a single letter there and allows subjects to infer that all of the letters are the same (Taylor, 2013).

iii. Prior knowledge.

The purpose of this paper is not to analyse (i) and (ii), as the new data pertain to (iii) (for more on (i) and (ii), see Taylor \& Sayim, 2018). To understand the significance of prior knowledge in the identity crowding debate, consider that when the stimuli are presented as they are in Figure 1 (and in Block's original paper) observers can (and usually will) look directly at the stimuli, and gain knowledge of what is there, which is prior to the judgements they make when fixating the + . In such a case, the ability to identify, discriminate, differentiate and think about the target does not imply that subjects could consciously see it. It may be that these abilities just reflect the fact that they have looked directly at it (and lack introspective capability to notice that while the stimulus looks like Ts, the actual number of Ts is less clear, see footnc 
Similar prior knowledge problems can also occur in experimental contexts. Telling you to identify the middle letter will inform you that there is a middle letter, from which you can infer that there are at least three items. Certain responses will then not be available to subjects. If you are told to identify the central letter, you are unlikely to reply with "there are only two letters there." Again, subjects' abilities to identify (discriminate, differentiate, think about) the target may then be based on-or biased by-this knowledge.

The hybrid view makes several empirically testable predictions. One is that when prior knowledge is eliminated, subjects will find it more difficult to identify, discriminate, differentiate and have thoughts about the middle letter. Below, we recount an experiment that did control for prior knowledge (Sayim \& Taylor, 2019). The data show that prior knowledge contributes very strongly to subjects' abilities in identity crowding. We argue that this is a problem for Block's claim that there is conscious seeing without attention in identity crowding. Specifically, we will argue that there is no reason to postulate conscious seeing without attention in these cases. We further argue that, since the hybrid view predicts this effect, the occurrence of the effect supports that view. We do not claim that the prior knowledge effects revealed in this paper are sufficient to constitute a response to Block on their own. As already indicated, prior knowledge functions only as part of the hybrid view.

\section{3 | THE MYSTERY OF THE MISSING LETTER}

\section{1 | The study}

First, a standard crowding experiment was performed that was subject to prior knowledge problems. In one condition, subjects were exposed to normal crowded stimuli (XTX). In the other, to identity crowded stimuli (TTT). Both sets of stimuli were embedded in blocks with numerous other sets of letters to avoid awareness of obvious repetitions. Subjects were informed that there were three letters there, and asked to report the middle one. In Experiment 1, subjects were much better in the identity crowded condition (94\% correct) as opposed to the normal crowded condition (46\% correct). Of course, this is predicted by the hybrid view, given prior knowledge problems.

In a second experiment, gaze-contingent presentation was used. This technique uses an eyetracker to record where the subjects are focussing their eyes. The stimuli only appeared when subjects were directly looking at the fixation point (similar to the "+" on the left in Figure 1). If subjects' eyes deviated from the fixation point, the stimuli were immediately removed from the screen before an eye movement to the stimulus could be completed. So it was impossible for subjects to focus their eyes directly on the stimuli. This eliminates the first element of the worry about prior knowledge (that subjects may look at the stimuli). Furthermore, the experimenter instructions were designed to give subjects as little information as possible. Subjects were asked to freely report and draw what they saw (for a similar drawing paradigm, see Coates, Wagemans, \& Sayim, 2017; Sayim \& Wagemans, 2017; Sayim, Myin, \& Van Uytven, 2015). In this way, subjects were given no hints about the kind or number of items there would be. This eliminates the second element of the prior knowledge worry (that task instructions may give away information).

Experiment 2 also had two conditions, one involving normal crowding, and the other involving identity crowding, with the same XTX and TTT stimuli as in the first experiment (the participants in the two experiments were different). When these constraints were put in place 
to eliminate prior knowledge, performance in the identity crowding condition plummeted, from 94\% correct in Experiment 1 to $44 \%$ in Experiment 2. All of the errors in the identity crowding case were caused by subjects mistaking the three items for two. That is, the errors came from mistaking "TTT" for "TT." This effect was observed both in the free verbal report measure as well as the drawings (experiments in which participants indicated the perceived location of and distance between the presented items suggest that the middle rather than one of the outer items goes missing in identity crowding, Yildirim, Coates, \& Sayim, 2019). Sayim and Taylor (2019) refer to this mistake as "redundancy masking" - a repeating, "redundant" item could not be reported because it was "masked" by the flankers (Yildirim, Coates, \& Sayim, 2020). Clearly, the data demonstrate that prior knowledge has a profound effect on performance in identity crowding, precisely as the hybrid view predicts.

We may ask what the subjects were attending in Experiment 2. The presentation times were as long as the subjects wanted, so they were free to attend whatever they could, including the group of letters as a whole, one or both of the flankers, and any part of these individual letters. However, for the sake of argument, we will can grant Block's claim that the target letter itself was unattended (2012).

In the redundancy masking condition of this experiment, only the letter $\mathrm{T}$ was used, but data from subsequent experiments demonstrates that redundancy masking also occurs with other shapes, such as lines (Yildirim et al., 2020), and more complex letters, indicating that it is not an effect unique to T's.

A third experiment by Sayim and Taylor provides some insight into the high success rate in Experiment 1 (where subjects were asked to identify the middle letter, and so had prior knowledge of the number of letters present). In the third experiment, subjects were given printouts of drawings, clearly depicting two letter T's, and allowed to look directly at them. They were asked what the (hypothetical) middle letter was, and if not sure, to take their best guess. A large majority of subjects reported ("guessed") "T" even though they knew that there was no middle letter, and their phenomenology would have been of two T's. This supplies us with additional reason to be highly sceptical of taking the fact that subjects can apparently "identify" (discriminate, differentiate, ...) a middle letter, and inferring that that their phenomenology contained three letters. After all, subjects will report a third letter even when we know that their phenomenology only represents two!

\section{2 | Interpreting the results}

We must distinguish our interpretation of Experiment 2 from its application to Block's original case. Take the experiment first. Clearly, the $66 \%$ of subjects who only reported two T's could not discriminate, differentiate, identify and have thoughts about the middle letter. So there is no reason to think they could consciously see the target. What about the $44 \%$ of times that the subjects correctly drew three T's? Could Block claim that in those cases the target was consciously seen? We do not claim that this view is inconsistent with the data. Of course it is possible that the subjects were consciously seeing the target. Rather, we claim that we do not have to accept it, as other explanations are available. For one thing, it is extremely difficult to plausibly make this case in the face of such a high error rate. ${ }^{2}$

Furthermore, prior knowledge is only one part of the hybrid view. Another element is macroscopic properties of the group (and flanker substitution). This aspect of Taylor and Sayim's hybrid view can adequately explain the $44 \%$ correct judgements of the participants. That is, it 
may be that in some cases subjects attended to the group itself, and were able to tell that the group was relatively congruous and uniform containing a repeating pattern. From this (and from the fact that they can consciously see at least one of the flankers) they can infer that any middle letter would be the same as the letter(s) that they can discern, have thoughts about it, and note that it is different from the background. ${ }^{3}$ This would then underpin their apparent ability to discern, discriminate, identify and have thoughts about the middle letter. We do not have to accept that the target was consciously seen, even in cases where subjects correctly reported three T's.

We must lay aside another important issue now. Note that in Experiment 2, performance increased in the normal crowding condition (up to 88\%). This is probably because of the increased exposure time compared to traditional crowding experiments, allowing a lot of time for examination of the stimuli. This high performance in the case of normal crowding raises important questions for the debate. Do we have good reason to think that the target was consciously seen? Did the target still escape attention, or did the increased exposure time allowed attention to "grab" the target item (in order for the "conscious seeing without attention" interpretation to hold up, this possibility would have to be excluded). These questions are important, but they will have to wait for future work, as this paper's focus is on identity crowding. One thing is for sure, however: the correctness rate in identity crowding is much lower than in the normal crowding case. This fits poorly with the widespread assumption that identity crowding functions as a better argument for conscious seeing without attention than normal crowding.

\section{4 | APPLYING THE RESULTS TO THE ORIGINAL CASE}

That concludes our interpretation of the experiment. How do these results apply to Block's original, informally presented case (2012)? This case is based on subjects' abilities to discriminate, differentiate, correctly identify and haye demonstrative thoughts about the target. In this case, prior knowledge effects are clearly present. We now know that prior knowledge dramatically increases subjects' (apparent) success in cases of identity crowding. From this, we can infer that it is highly likely that a large part of the apparent ability to identify, discriminate, differentiate and think about the central letter in Block's original 2012 paper can be explained in terms of prior knowledge, rather than conscious perception of the target.

Again, this is not to say that other factors (like grouping or congruity effects) do not also play a role. The hybrid view would say that subjects have prior knowledge of the target, and the overall congruous appearance of the stimuli, or mistaking one of the flankers for the middle letter, serve to confirm this prior knowledge. They may then use a combination of this information to judge that there is a middle letter and also to identify it. This constitutes an adequate explanation of our apparent ability to identify, discriminate and differentiate the middle letter in Block's original (2012) case.

What about the claim that we have demonstrative thoughts about the middle letter (Block, 2012, p. 177)? On the hybrid view, subjects infer the presence of a middle letter (in the way explained above) and this information forms the basis of their ability to have thoughts about it (Taylor, 2013). An opponent could object that thoughts based on inference do not count as demonstrative, because they are not based on singling out the item in conscious vision. However, we simply deny that we have demonstrative thoughts about the middle letter in this sense. We are entitled to conclude that we are able to have thoughts about the middle letter, which is something that the hybrid view can explain. We are not entitled to claim that these thoughts 
are demonstrative in the sense of being based on conscious singling out of the item in vision (indeed, to assume this would be to beg the question in favour of Block's interpretation, as it assumes that the middle letter features in consciousness).

We can also draw a much more general lesson. In the original 2012 case, all of the information subjects need for the abilities in question is already available to the subject through prior knowledge. Ultimately, whenever prior knowledge is present to such a strong degree, we cannot ever be confident drawing conclusions about conscious seeing from subjects' apparent abilities.

An opponent may reply that, in Block's, 2012 paper, subjects' prior knowledge helps them to consciously see the target. ${ }^{4}$ That is, while they are attending the + on the left, they can use the prior knowledge to construct a conscious perception of the middle letter, either directly (though cognitive penetration), or by altering patterns of attention to the stimuli, which in turn has an impact on the phenomenology of perception (Firestone \& Scholl, 2015; Pylyshyn, 1999). In response, we do not claim that the experiments prove that subjects did not consciously see the middle letter in Block's cases. Our claim is rather that we do not have good reason to think that they consciously see it, because all of the abilities in question can be accounted for in other terms. Unless we have additional reason to believe that prior knowledge is affecting subjects in this way, then the conscious seeing without attention view will not be established.

An opponent may appeal directly to introspection, claiming that when we introspect our experience, we find that we can discern, differentiate, identify and have demonstrative thoughts about the target, in a way that is clearly based on conscious perception of it. ${ }^{5}$ The problem with this suggestion is that in this debate, the phenomenology is simply too controversial to be appealed to in this way. Whether the phenomenology includes the middle letter is precisely what is at issue. The results of our introspection may be theory-laden (and hence untrustworthy). Furthermore, the result of such an argument would most likely be dialectical deadlock over the deliverances of introspection. This is not a promising route.

\section{5 | OTHER VIEWS}

Here we briefly review the relevance of the results for three other positions in the debate.

\section{1 | Michael Tye}

Tye mentions prior knowledge problems, suggesting that if you are "primed" to see three letters, then that is what you will report $(2014$, p. 157). This is precisely what was found in the experiment recounted above. The results are less congenial to other aspects of Tye's view, though. He claims that with identity crowding, we have a conscious experience of two T's, not three $(2014$, p. 156). Returning to Experiment 2, this may be a plausible explanation of the $66 \%$ of cases where subjects reported two T's, but it struggles to explain the $44 \%$ of correct responses. If subjects simply saw two T's, they would have drawn two T's, not three. This does not show that his view is false, just incomplete (he could of course invoke some of the factors that the hybrid view uses to explain the $44 \%$ of correct guesses, but we leave it to Tye to modify his position).

Tye's argument for his view is also problematic. He recommends fixating on the three letters, then switching your fixation point to the "+" on the left. He claims that when you do this, one of the letters simply disappears from your consciousness. The problem is that being asked 
to focus on the letters and then switch our gaze to the " knowledge of the stimuli*** (Block, 2014 also criticises Ty isures that you will have prior thod). This immediately raises the familiar difficulty of disentangling prior knowledge from conscious seeing. Therefore, the method is not trustworthy.

\section{2 | Bradley Richards and Adrienne Prettyman}

Richards $(2013,2016)$ suggests that we unconsciously see the central letter, and this triggers a conscious thought about its identity. Prettyman (2018) claims that we can see all of the letters in the stimuli, and that we do not see the target as an object in its own right, but as a local part of a global object. This global object is itself attended. The new data demonstrate a lacuna in both Richards' and Prettyman's view. Neither of them give prior knowledge a prominent role in their accounts. Since we now know that prior knowledge is such an important factor in explaining identity crowding, these views will be obliged to incorporate these effects in their explanations.

Here it is worth raising a more direct issue with Prettyman's view. The extent to which perceiving the target as part of a global object (i.e., the extent to which grouping of the parts into larger wholes), plays a role in crowding has been the topic of a large number of studies (Banks \& White, 1984; Manassi, Sayim, \& Herzog, 2012, 2013; Overvliet \& Sayim, 2015; Prinzmetal \& Banks, 1977; Saarela, Sayim, Westheimer, \& Herzog, 2009; Sayim \& Cavanagh, 2013; Sayim, Greenwood, \& Cavanagh, 2014; Sayim, Westheimer, \& Herzog, 2008, 2010, 2011; Taylor \& Sayim, 2018). Prettyman argues that the target is seen as part of a global object, and that we consciously see the middle letter as part of this global object. But this sits uncomfortably with the fact that strong target-flanker grouping into a global object decreases subjects' abilities to identify the target item (see Herzog, Sayim, Chicherov, \& Manassi, 2015 for a review; but see Melnik, Coates, \& Sayim, 2018, 2020; Rummens \& Sayim, 2019). So if the target is indeed grouping strongly into a global object with the flankers, we would actually have less reason to think that the target is consciously seen.

\section{6 | CONCLUSION}

We have reviewed evidence that prior knowledge has a dramatic effect on performance in identity crowding, which is vividly illustrated through the redundancy masking effect. This supports views (such as the hybrid view) which invoke prior knowledge in their explanations, and are problematic for views that claim we consciously see the target item.

\section{ORCID}

(John) Henry Taylor (D https://orcid.org/0000-0001-7504-0283

\section{ENDNOTES}

${ }^{1}$ Though he allows that there may be spatial attention to the region (2012).

${ }^{2}$ Note that it is consistent with the data that subjects saw "a group of T's", which is indeterminate over the precise number. 
${ }^{3}$ The fact that there is a middle letter may be provided by the fact that the group appears "cluttered" (see Taylor, 2013).

${ }^{4}$ Thanks to an anonymous referee.

${ }^{5}$ Thanks to an anonymous referee for this. Block may be interpreted as expressing something like this when he emphasises that our abilities with relation to the identity crowded stimuli are conscious abilities (Block, 2012, p. 175).

\section{REFERENCES}

Banks, W. P., \& White, H. (1984). Lateral interference and perceptual grouping in visual detection. Perception \& Psychophysics, 36(3), 285-295. https://doi.org/10.3758/BF03206370

Block, N. (2012). The grain of vision and the grain of attention. Thought, 1, 170-184.

Block, N. (2013). Seeing and windows of integration. Thought, 2(1), 29-39.

Block, N. (2014). The defective armchair: A reply to Tye. Thought, 3(2), 159-165.

Cavanagh, P., He, S., \& Intriligator, J. (1999). Attentional resolution: The grain and locus of visual awareness. In C. Taddei-Ferretti \& C. Musio (Eds.), Neuronal basis and psychological aspects of consciousness. Singapore: World Scientific.

Coates, D. R., Wagemans, J., \& Sayim, B. (2017). Diagnosing the periphery: Using the Rey-Osterrieth complex figure drawing test to characterize peripheral visual function. Perception, 8(3), 1-20. https://doi.org/10.1177/ 2041669517705447

Firestone, C., \& Scholl, B. (2015). Cognition does not affect perception: Evaluating the evidence for 'top-down' effects. Behavioral and Brain Sciences, 39, e229. https://doi.org/10.1017/S0140525X15000965

Freeman, J., \& Pelli, D. (2007). An escape from crowding. Journal of Vision, 7, 1-14.

Greenwood, J. A., Szinte, M., Sayim, B., \& Cavanagh, P. (2017). Variations in crowding, saccadic precision, and spatial localization reveal the shared topology of spatial vision. Proceedings of the National Academy of Sciences of the United States of America, E3573-E3582. https://doi.org/10.1073/pnas.1615504114

Herzog, M. H., Sayim, B., Chicherov, V., \& Manassi, M. (2015). Crowding, grouping, and object recognition: A matter of appearance. Journal of Vision, 15(6), 5-5. https://doi.org/10.1167/15.6.5

Levi, D. (2008). Crowding-an essential bottleneck for object recognition: A mini review. Vision Research, 48, 635-654.

Manassi, M., Sayim, B., \& Herzog, M. H. (2012). Grouping, pooling, and when bigger is better in visual crowding. Journal of Vision, 12(10), 1-14. https://doi.org/10.1167/12.10.13

Manassi, M., Sayim, B., \& Herzog, M. H. (2013). When crowding of crowding leads to uncrowding. Journal of Vision, 13(13), 1-10. https://doi.org/10.1167/13.13.10

Melnik, N., Coates, D. R., \& Sayim, B. (2018). Emergent features in the crowding zone: When target-flanker grouping surmounts crowding. Journal of Vision, 18-19(9), 1-15. https://doi.org/10.1167/18.9.19. http://jov. arvojournals.org/article. aspx?articleid $=2703422$

Melnik, N., Coates, D. R., \& Sayim, B. (2020). Emergent features break the rules of crowding. Scientific Reports, 10, 406. https://doi.org/10.1038/s41598-019-57277-y

Overvliet, K. E., \& Sayim, B. (2015). Perceptual grouping determines haptic contextual modulation. Vision Research in press.

Petrov, Y., \& Meleshkevich, O. (2011). Asymmetries and idiosyncratic hot spots in crowding. Vision Research, 51 Q8 (10), 1117-1123. https://doi.org/10.1016/j.visres.2011.03.001

Prettyman, A. (2018). Seeing the forest and the trees: A response to the identity crowding debate. Thought, 7 , $30-40$.

Prinzmetal, W., \& Banks, W. P. (1977). Good continuation affects visual detection. Perception \& Psychophysics, 21 (5), 389-395. https://doi.org/10.3758/BF03199491

Pylyshyn, Z. (1999). Is vision continuous with cognition? Behavioral and Brain Sciences, 22, 341-423.

Richards, B. (2013). Identity crowding and object seeing: A reply to Block. Thought, 2, 9-19.

Richards, B. (2016). Attention and seeing objects: The identity crowding debate. Philosophical Psychology, 29, 743-758. 
Rummens, K., \& Sayim, B. (2019). Disrupting uniformity: Feature contrasts that reduce crowding interfere with peripheral word recognition. Vision Research, 161, 25-35.

Saarela, T. P., Sayim, B., Westheimer, G., \& Herzog, M. H. (2009). Global stimulus configuration modulates crowding. Journal of Vision, 9(2), 1-11. https://doi.org/10.1167/9.2.5

Sayim, B., \& Cavanagh, P. (2013). Grouping and crowding affect target appearance over different spatial scales. PLoS One, 8(8), e71188. https://doi.org/10.1371/journal.pone.0071188

Sayim, B., Greenwood, J. A., \& Cavanagh, P. (2014). Foveal target repetitions reduce crowding. Journal of Vision, 14(6), 1-12. https://doi.org/10.1167/14.6.4

Sayim, B., Myin, E., \& Van Uytven, T. (2015). Prior knowledge modulates peripheral color appearance. Proceedings of the International Colour Association (AIC), Tokyo Midterm meeting, 2015 (pp. 1147-1152).

Sayim, B., \& Taylor, H. (2019). Letters lost: Capturing appearance in crowded peripheral vision reveals a new kind of masking. Psychological Science, 30, 1082-1086. https://doi.org/10.1177/0956797619847166

Sayim, B., \& Wagemans, J. (2017). Appearance changes and error characteristics in crowding revealed by drawings. Journal of Vision, 17(11), 1-16. https://doi.org/10.1167/17.11.8

Sayim, B., Westheimer, G., \& Herzog, M. H. (2008). Contrast polarity, chromaticity, and stereoscopic depth modulate contextual interactions in vernier acuity. Journal of Vision, 8(8), 1-9. https://doi.org/10.1167/8.8.12

Sayim, B., Westheimer, G., \& Herzog, M. H. (2010). Gestalt factors modulate basic spatial vision. Psychological Science, 21(5), 641-644. https://doi.org/10.1177/0956797610368811

Sayim, B., Westheimer, G., \& Herzog, M. H. (2011). Quantifying target conspicuity in contextual modulation by visual search. Journal of Vision, 11(1), 1-11.

Taylor, H. (2013). Is the grain of vision finer than the grain of attention? Response to Block. Thought, 2, $20-28$.

Taylor, H. (2018). Attention, psychology and pluralism. The British Journal for the Philosophy of Science, 69, 935-956.

Taylor, H., \& Sayim, B. (2018). Crowding, attention and consciousness: In support of the inference hypothesis. Mind and Language, 33, 17-33.

Toet, A., \& Levi, D. M. (1992). The two-dimensional shape of spatial interaction zones in the parafovea. Vision Research, 32(7), 1349-1357. https://doi.org/10.1016/0042-6989(92)90227-A

Tye, M. (2014). Does conscious seeing have a finer grain than attention? Thought, 3, 154-158.

Yildirim, F. Z., Coates, D. R., \& Sayim, B. (2019). Lost lines in warped space: Evidence for spatial compression in crowded displays. Annual Meeting of the Vision Science Society, May 2019, St Pete Beach, USA (pp. 13-13).

Yildirim, F. Z., Coates, D. R., \& Sayim, B. (2020). Redundancy masking: The loss of repeated items in crowded peripheral vision. Journal of Vision in press.

How to cite this article: Taylor (John) Henry, Sayim B. Redundancy masking and the identity crowding debate. Thought: A Journal of Philosophy. 2020;1-9. https://doi.org/10. $\underline{1002 / \text { tht } 3.469}$ 